\section{Statins and the COVID-19 Vaccines: a viewpoint}

\section{DOI: 10.52629/jamsa.v9i1.253}

Abstract The COVID-19 pandemic has caused many scientists to promptly make vaccines against the disease. A large number of people who will receive the vaccines are likely to be older adults, many of them being long-term statin users. Statins, known to be immunomodulators, can interact with vaccines and alter their effects, like in the case of the influenza vaccine, whether this effect is significant or not is up for debate. Thus, the interaction between statins and the COVID19 vaccines should be investigated and understood, and guidelines should be written to ensure that the vaccines perform optimally.

Keywords COVID-19; Vaccine; Effectiveness; Statins; Influenza; Immunomodulatory
Muhammad Sameer

Arshad', Asad Ali Siddiqui'

1 Department of Medicine, Dow University of Health Sciences,

Karachi, Pakistan
Correspondence to: Muhammad Sameer Arshad Department of Medicine, Dow University of Health Sciences, Karachi, Pakistan

Muhammadsameerarshad@ gmail.com 


\section{Introduction}

Severe acute respiratory syndrome coronavirus 2 (SARS-CoV-2) vaccines have already been developed by various drug makers including Moderna, BioNTech/Pfizer, Johnson \& Johnson, Sanofi, Oxford/AstraZeneca, and GlaxoSmithKline. At the time of writing this, these companies are rolling out their vaccines worldwide to people belonging to different races, ethnicities, social classes, and age groups. Therefore, it is quite likely that people who are currently under some sort of medication would also opt to receive these vaccines. In this case, the vaccine may interact with the drug(s) in the recipient's system, leading to altered vaccine effectiveness (VE). One such drug class that may lead to an altered $V E$ is the statins.

\section{Statins}

Statins are one of the most widely prescribed drugs in the world. In the US, approximately $71 \%$ of adults with diagnosed cardiovascular disease, 63\% of those with diagnosed diabetes, and $54 \%$ of those with

diagnosed hypercholesterolemia reported taking prescription cholesterol-lowering medications, $93 \%$ of which consisted of statins ${ }^{1} \quad$ These drugs treat hypercholesterolemia by inhibiting the HMG-CoA reductase enzyme, thus reducing the risk of cardiovascular disease, especially in older patients. Statins are also known to have immunomodulatory effects, and studies have even shown that these drugs can reduce the expression of major histocompatibility complex II (MHC-
II) ${ }^{2}$, thereby making it possible to reduce $V E$ by reducing the adaptive immune response. One need not look any further than the vaccine for influenza to assess the effect of statins.

\section{Statins and Influenza}

Various studies have been conducted that judge the relationship between statin usage and vaccine response. In a post hoc analysis by Black et al, it was shown that hemagglutination-inhibiting geometric mean titers to influenza $A(H 1 N 1), A(H 3 N 2)$, and $B$ strains were lower (by about 38\%) in patients who had been receiving long term statin therapy ${ }^{3}$. In a retrospective cohort study by Omer et $\mathrm{al}^{4}$ over nine influenza seasons between 2002 and 2011, after adjusting for incidence when influenza was not widespread, it was shown that statin therapy was associated with reduced influenza VE against medically attended acute respiratory illness (MAARI). Therefore, these studies showed that chronic statin usage can reduce influenza VE.

However, other studies have shown that there was no significant change in vaccine response. In a study by Havers et al, patients aged $\geq 45$ years with MAARI over 6 influenza seasons were assessed. Amongst the patients, 30\% were statin users and $24 \%$ tested positive for influenza virus infection, while $78 \%$ of statin users and $60 \%$ of nonusers had received influenza vaccine. It was shown that VE against influenza $\mathrm{A}(\mathrm{H} 1 \mathrm{~N} 1)$ pdm09, $\mathrm{A}(\mathrm{H} 3 \mathrm{~N} 2)$, and $B$ viruses were similar amongst statin users and nonusers 5 . Additionally, some studies have shown more mixed results. In 
a multi-year study by Mclean et al, 3285 adults were investigated, out of which $37 \%$ were statin nonusers, $27 \%$ were unvaccinated statin nonusers, $26 \%$ were vaccinated statin users, and 10\% were unvaccinated statin users - thus making a total of $100 \%$. Their results showed that statin use was associated with reduced $V E$ against influenza $A(H 3 N 2)$ but not influenza $A(\mathrm{H} 1 \mathrm{~N} 1)$ pdm09 or influenza $B^{6}$.

\section{Why It Matters}

We admit that SARS-CoV-2 and the influenza viruses have different structures and mechanisms of action. The same can also be said for their vaccines. However, the point we are trying to highlight is that, if there is a diminished effect observed in one vaccine amongst a particular group of people, is it not possible to observe the same in another vaccine for the same group?

The reason why influenza is chosen and discussed is because the influenza vaccine has the most extensive literature, with clinical trials that also focus on the effect of statins, unlike trials for other viral vaccines. However, despite there being many trials focused on this sub-populace, there is still great confusion regarding influenza VE in statin users . Of the people who are diagnosed with COVID-19, it is the elderly that show more severe symptoms and have higher mortality rates ${ }^{7}$. They are thus more in need of the vaccine relative to other subpopulations. When a literature search was done on the COVID-19 vaccine trials, we found no investigation on statin users, let alone those in older age groups. We feel that this gap in literature should be addressed to ensure optimal utilization of the vaccine.

Older adults are prescribed various drugs due to their frailty and relatively weak immune systems ${ }^{7}$. One such drug that is very commonly given is statins. In the US alone, during 2011-2012, around one in four adults aged 40 or above reported using a statin, either by itself, or in combination with another cholesterol-lowering medication, and it is only likely that this number has risen'. It is therefore important to investigate the interactions of statins both synthetic and natural - with the COVID-19 vaccine. Studies should be designed in a way such that they could determine whether statin usage reduces the immunogenicity of the COVID-19 vaccine, and if so, by what mechanism(s).

In case there is a reduced VE, future studies could then explore if this diminished effect is clinically significant, and different strategies for administering the vaccine to overcome this issue could be devised based on the results. Temporarily halting the prescription of statins to populations at risk before giving the vaccine, administering higher dosages of the vaccine, developing and using adjuvants to improve immunogenicity, and alternate injection sites (such as intradermal injections) could all be explored. The intrinsic side effects of statins and the patient's physical condition should also be considered when deciding what approach to take when vaccinating. Most importantly, the physician should remain up to date with all the literature regarding this issue to make a more informed decision. 


\section{Conclusion}

Developing and distributing the vaccine to the masses is an expensive and timeconsuming affair. There is a very high demand for the vaccine, and it would not be easy for manufacturers to maintain supply. It is unlikely that everyone who wants or even needs the vaccine would get it. Therefore, those who do receive the vaccine should derive the maximum benefit from it, making no compromises on its effectiveness. There should therefore be no confusion regarding the relationship between statins and the COVID-19 vaccines, as there is with influenza vaccines. By prioritizing observational and experimental studies on this matter, researchers could help establish clear guidelines for physicians to follow, thus saving many valuable lives.

\section{References}

1. Gu Q, Paulose-Ram R, Burt VL, Kit BK. Prescription cholesterol-lowering medication use in adults aged 40 and over: United States, 2003-2012. NCHS Data Brief. 2014;(177):1-8.

2. Terblanche M, Almog Y, Rosenson RS, Smith TS, Hackam DG. Statins and sepsis: multiple modifications at multiple levels. Lancet Infect Dis. 2007;7(5):358-368. doi:10.1016/S1473-3099(07)70111-1

3. Black S, Nicolay U, Del Giudice G, Rappuoli R. Influence of Statins on Influenza Vaccine Response in Elderly Individuals. J Infect Dis. 2016;213(8):1224-1228.

doi:10.1093/infdis/jiv456

4. Omer SB, Phadke VK, Bednarczyk RA, Chamberlain AT, Brosseau JL, Orenstein WA. Impact of Statins on Influenza Vaccine Effectiveness Against Medically Attended Acute Respiratory Illness. J Infect Dis.
2016;213(8):1216-1223.

doi:10.1093/infdis/jiv457

5. Havers FP, Chung JR, Belongia EA, et al. Influenza vaccine effectiveness and statin use among adults in the United States, 20112017. Clin Infect Dis. 2019;68(10):1616-1622. doi:10.1093/cid/ciy780

6. McLean HQ, Chow BDW, Vanwormer JJ, King JP, Belongia EA. Effect of Statin Use on Influenza Vaccine Effectiveness. J Infect Dis. 2016;214(8):1150-1158. doi:10.1093/infdis/jiw335

7. Kadambari S, Klenerman P, Pollard AJ. Why the elderly appear to be more severely affected by COVID-19: The potential role of immunosenescence and CMV. Rev Med Virol. 2020;30(5). doi:10.1002/rmv.2144 